\title{
Damping in magnetorheological elastomers under compressive stress
}

\author{
Mateusz Kukla ${ }^{1 *}$, Krzysztof Talaśka ${ }^{1}$, and Ireneusz Malujda ${ }^{1}$ \\ ${ }^{1}$ Poznan University of Technology, Chair of Basics of Machine Design, 3 Piotrowo St., \\ 60-965 Poznań, Poland
}

\begin{abstract}
Magnetorheological elastomers are an important area of study in non-classical engineering materials. These are smart materials, in which some of the physical properties are dependent on the applied magnetic field. This unique property allows to suggest new, innovative practical applications. It is therefore relevant to carry out studies in the possible application of magnetorheological elastomers in machine construction. The present article presents the results of study regarding the properties of the discussed materials subject to compressive stresses. Particular attention is given to the observed growth of surface area of mechanical hysteresis loops, which is evidence of the possibility to change the damping properties of magnetorheological elastomers. This property can be utilized in the construction of different types of machines and devices. These mostly applies to energy absorbers such as active vibration absorbers.
\end{abstract}

Keywords: magnetorheological elastomers, compressive stresses, relative damping, storage modulus, loss modulus

\section{Introduction}

Magnetorheological elastomers have a broad application in vibration absorption or damping systems. The use of materials able to change their properties under external conditions opens up unprecedented possibilities. It allows to construct a vibration absorber with variable characteristics, depending on the applied magnetic field. A system utilizing such device would be able to change its resonant frequency. An appropriate control algorithm may adjust the damping value in real time, within a certain range, to match the excitation frequency. Recently, an increase in the number of suggested applications for the utilization of magnetorheological elastomers in these types of systems is observed. Such applications include active linear vibration dampers which utilize the discussed materials, in compression mode [1] or shear mode [2,3] together with active torsion vibration dampers $[4,5,6]$. As a continuation of previous study [7] to identify the possibility to utilize magnetorheological elastomers in machine construction, a specialized testing station was

\footnotetext{
${ }^{*}$ Corresponding author. Tel.: +48 612244514

E-mail address: mateusz.kukla@put.poznan.pl

Reviewers: Vladimir Dekýš, Robert Zalewski
} 
designed. This allowed to verify the capabilities of the composite samples to absorb and dissipate mechanical energy.

\section{Testing station}

The concept of the designed system assumed the utilization of an electro-hydraulic servomechanism with displacement error adjustment. Despite the degree of complexity of such a solution, it was characterized by a number of advantages. The major advantage is the possibility to set the displacement profile of the servomechanism piston and to execute it with the possibly lowest degree of error. The specialized testing station consists of: mechanicalhydraulic working system, electrical control system and a data registration and acquisition system. System diagram is shown on Fig. 1.

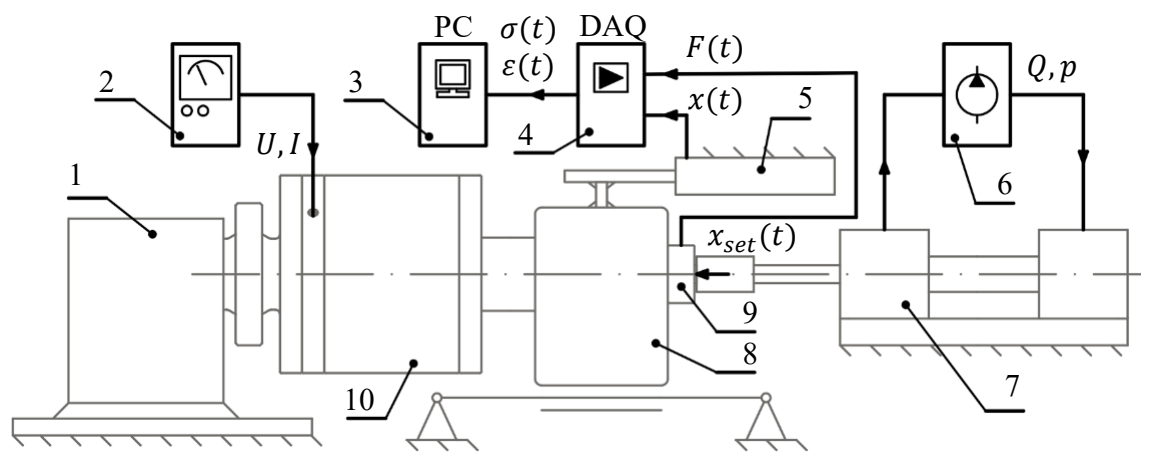

Fig. 1. Diagram of the specialized testing station; 1 - base, 2 - laboratory power supply unit, 3 - PC, 4 - data acquisition system (DAQ), 5 - displacement sensor, 6 - hydraulic system, 7 -hydraulic servo, 8 - guidance with load transfer component, 9 - force sensor, 10 - measuring system fitted with a magnetorheological elastomer sample

Servomotor 7 is connected to the hydraulic system 6 , which is characterized by the parameters: volumetric flow rate $Q$ and pressure $p$. It generates an input function (displacement) as per the control system setting. Piston displacement is transferred, via the intermediary component 8 fitted with linear guidance to measuring unit 10 rigidly connected to the base 1 . The tested magnetorheological elastomer sample is placed inside the measuring system. The induction coil of the system is powered by direct current characterized by voltage $U$ and current $I$ value as provided by the laboratory power supply unit 2 . Force value measurement is carried out via force sensor 9 (C9C $1 \mathrm{kN}$ by HBM). The linear displacement sensor 5 (WA $20 \mathrm{~mm}$ by HBM) is utilized for measuring displacement value. Signals from both sensors are transferred to auxiliary amplifier 4 working together with a PC unit 3, equipped with data acquisition software. Based on the measurement result of compression force and displacement of servomotor piston and the intermediary component (directly causing the compression of elastomer sample), stress and strain values are calculated. Fig. 2 shows the assembly of the mechanical testing station. The electrical diagram of the control system is shown on Fig. 3. 


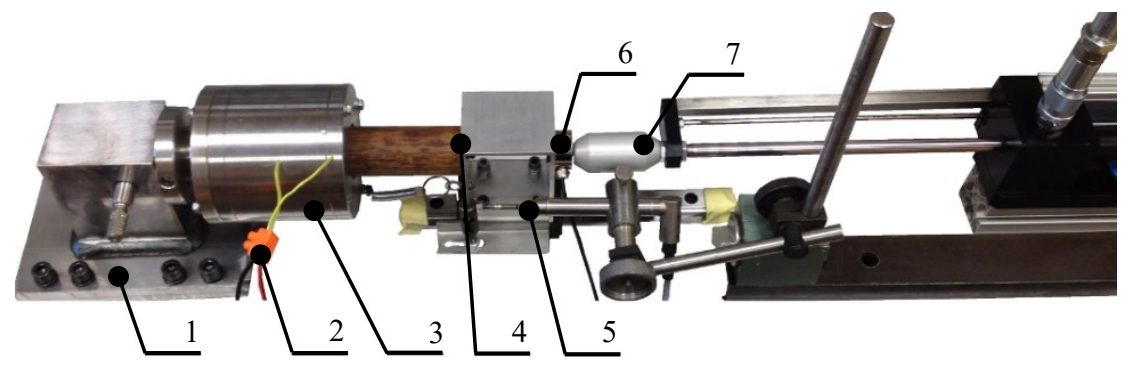

Fig. 2. View of the mechanical testing station assembly; 1 - base, 2 - power supply, 3 - measuring assembly with magnetorheological elastomer sample, 4 - guidance with load transfer component, 5 - displacement sensor, 6 - force sensor, 7 - hydraulic servo

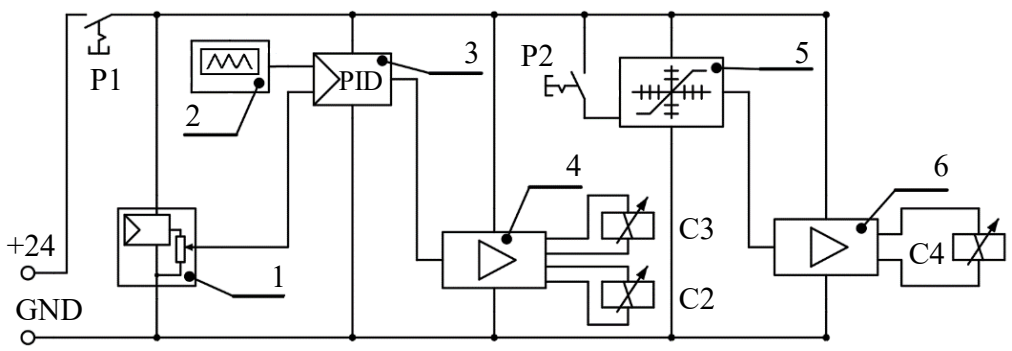

Fig. 3. Electrical diagram of the testing station control system; 1 - displacement sensor, 2 - laboratory signal generator, 3 -PID controller, 4 - dual channel proportional amplifier, 5 - parameter setting card, 6 - single channel proportional amplifier

The laboratory signal generator 2 provides the control signal transmitted to the PID controller 3. The displacement sensor, rigidly attached to the servo piston rod, works together with the controller within a negative feedback loop. The control signal is transmitted from the regulator to the dual channel proportional amplifier 4, which controls the coils C3 and C2 in the electro-hydraulic servo valve. The parameter setting card 5 allows to generate a constant signal with adjustable value. It is transmitted to the single channel proportional amplifier 6, which controls the coil $\mathrm{C} 4$ of the over-flow valve. This allows accurate control of the pressure value in the system. The control system is activated with button P1, whereas the hydraulic system is activated with button P2. The hydraulic diagram of the working system is provided on Fig. 4.

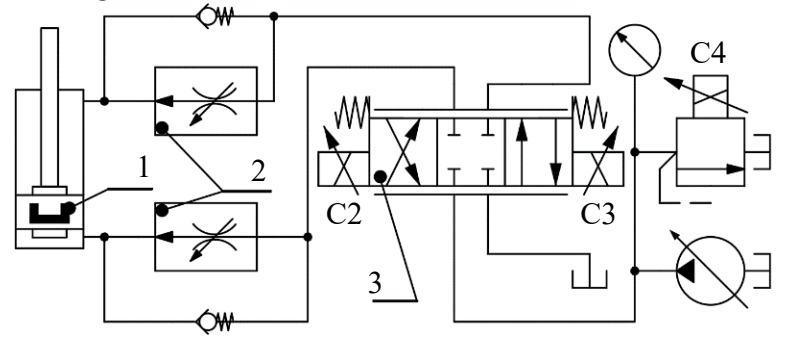

Fig. 4. Hydraulic diagram of the working system of the testing station; 1 - displacement sensor (built into the sensor), 2 - flow regulators, 3 - electrohydraulic servo valve

The electrohydraulic servo valve 3 is working together with flow regulators 2 which are connected to the servo. Such a solution allows, with an appropriate setting of control parameters, to set the piston rod retraction speed. It remains constant regardless of the force 
to be overcome by the servo (the compressive force of the tested sample). The coils in proportional components $\mathrm{C} 2-\mathrm{C} 4$ are powered by signals from the amplifiers as provided on Fig. 3. The general view of the specialized testing station is provided on Fig. 5.

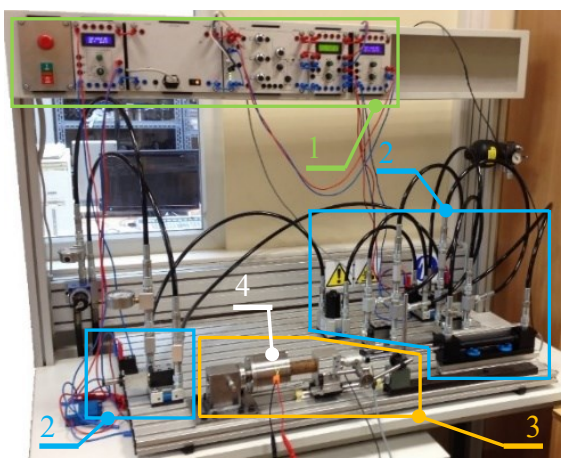

Fig. 5. General view of the specialized testing station; 1 - electrical control system, 2 - hydraulic working unit, 3 - mechanical unit, 4 - magnetorheological elastomer (inside the coil)

\section{Testing methodology}

Four samples of magnetorheological elastomers with no difference in composition or method of manufacturing. Polyurethane, being a mixture of polyols VORALUX® HF 505 and 14922 isocyanate HB 6013, was used as matrix. Polyurethane polymeric materials are widely used in the machine design [8]. The employed ferromagnetic material is powdered carbonyl iron with particle diameter in the range of 6 to $9 \mu \mathrm{m}$. The constituent materials were mixed mechanically, gas stripped and placed in a mold. Crosslinking took place in the presence of magnetic field with magnetic induction of $B=300 \mathrm{mT}$. The shape of the tested samples are derived from mold geometry, i.e. cylinders with diameter $d=20 \mathrm{~mm}$ and height $h=20 \mathrm{~mm}$. Two different strain amplitudes $\varepsilon_{0}$ for four different frequency variants $f$ were selected for testing. By controlling the strain as a triangular waveform function (which characterized the servo piston displacement), the magnetorheological elastomer sample was subject to compressive stress (caused by compressive force generated by the servo piston). The sample was first stabilized and subsequently, ten full cycles of applying and removing the load, thus achieving $m=10$ hysteresis loops for the single tested sample, which were subsequently used to calculated the average value. The measurements were taken for different induction values of the magnetic field, in a sequence: $B=0 \mathrm{mT}, 64 \mathrm{mT}, 127 \mathrm{mT}$. The diagram of the testing program is provided on Fig. 6 . The strain amplitude value $\varepsilon_{0}$ and frequency $f$ used in the study were based on the characteristics of the designed testing station. Accurate execution of the set speed parameter value and displacement in time progression depended on the pressure value $\mathrm{p}$ and the volumetric flow current $Q$ of oil in the hydraulic system, the effective change thereof was possible within a particular range. 


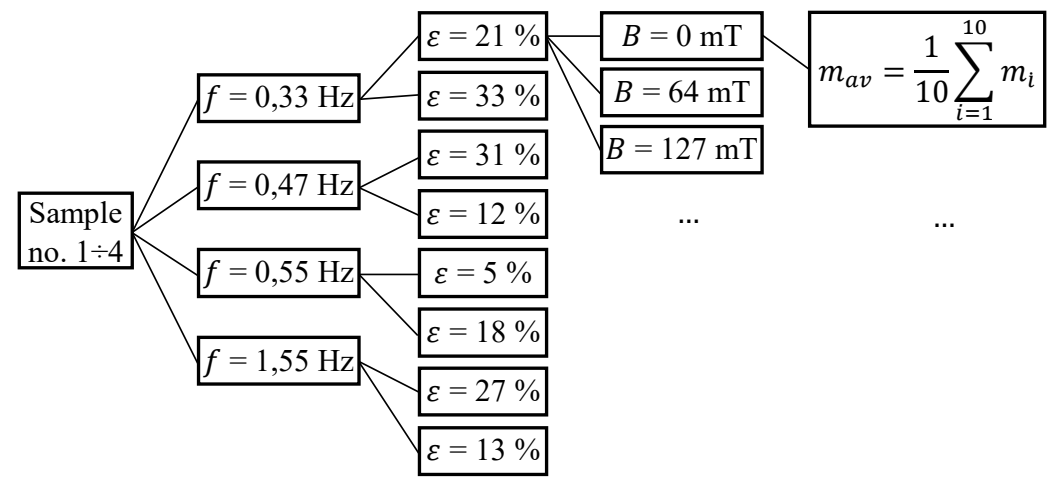

Fig. 6. Diagram of the performed testing program

Based on the obtained results, a number of material parameters were established allowing to evaluate the changes in the tested composite subject to a magnetic field. To this end, the Kelvin-Voigt rheological model, often used to analyze materials of this group [9], was employed. The structure of this model is based on two modulus values describing the material's ability to gather and dissipate energy. These are: storage modulus $E^{\prime}$ (elasticity) and loss modulus $E^{\prime \prime}$ (viscosity). These were established based on the characteristic points in the measured hysteresis loop. Additionally, the value of dissipated energy was also established $W$ (damping work) and the value of elastic energy $\Delta W$ (strain work) in order to determine the change of relative damping $\psi$. The provided physical values can be calculated from the hysteresis loop surface area (Fig. 7) in the loading-unloading cycle.

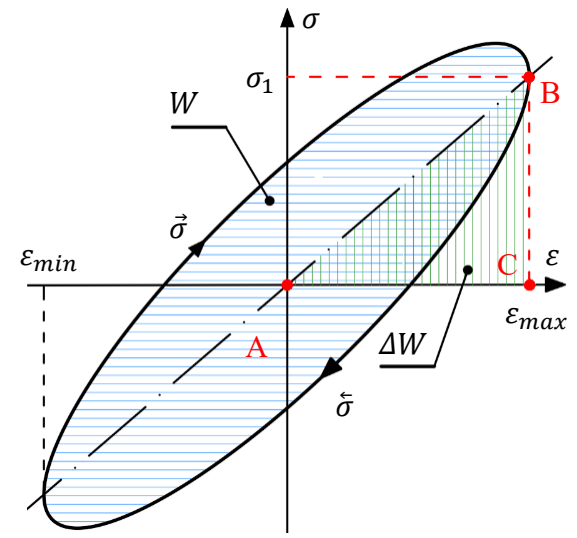

Fig. 7. A hysteresis loop schematic in the strain-stress cycle for the stabilized sample; based on [10]

The dissipated energy value is interpreted as the entire hysteresis loop surface area $W$ (horizontally hatched area). The elastic energy value $\Delta W$ is the area of the $\mathrm{A}, \mathrm{B}, \mathrm{C}$ triangle (vertically hatched area). It is defined by three sections. These are: the $\mathrm{AB}$ section connecting the minimum and maximum strain value (intersecting the origin), the $\mathrm{BC}$ section parallel to the stress axis, connecting the maximum strain with the corresponding point on the hysteresis loop and part of the strain axis completing the triangle, defined as section AC. Based on the above values, it is possible to calculate relative damping $\psi$. This coefficient is one of the indices for energy dissipation. The values can be derived from the following formulas: 


$$
\begin{gathered}
W=\int_{\varepsilon_{\min }}^{\varepsilon_{\max }} \vec{\sigma} d \varepsilon-\int_{\varepsilon_{\max }}^{\varepsilon_{\min }} \overleftarrow{\sigma} d \varepsilon, \\
\Delta W=\frac{\sigma_{1}}{\varepsilon_{\max }} \int_{0}^{\varepsilon_{\max }} \varepsilon d \varepsilon \\
\psi=\frac{\Delta W}{W}
\end{gathered}
$$

where: $\varepsilon_{\min }$ - minimum strain value, $\varepsilon_{\max }$ - maximum strain value, $\vec{\sigma}$ - change of stress in the range from $\varepsilon_{\min }$ to $\varepsilon_{\max }, \overleftarrow{\sigma}$ - change of stress in the range from $\varepsilon_{\max }$ to $\varepsilon_{\min }, \sigma_{1}-$ stress corresponding to strain $\varepsilon_{\max }$.

\section{Study results}

The set of all the recorded hysteresis loops is provided on Fig. 8. There is a visible increase in the angle of inclination of the diagonals and surface area of the obtained hysteresis loops as a function of magnetic field induction. The analysis of presented characteristics leads to a conclusion that the change of maximum strain of the tested sample causes change of edge of the hysteresis loop. The higher the maximum stress, the more difference between the resultant progression from a perfect elliptical shape. The individual parts of the hysteresis loop are characterized by an increasing progression, whereas the growth of stress becomes less linear in relation to strain. This results from the fact that an increase in strain causes an increase in the distribution of stress. This is caused by high susceptibility of the mold material which causes significant alteration in the sample cross section as a result of load.

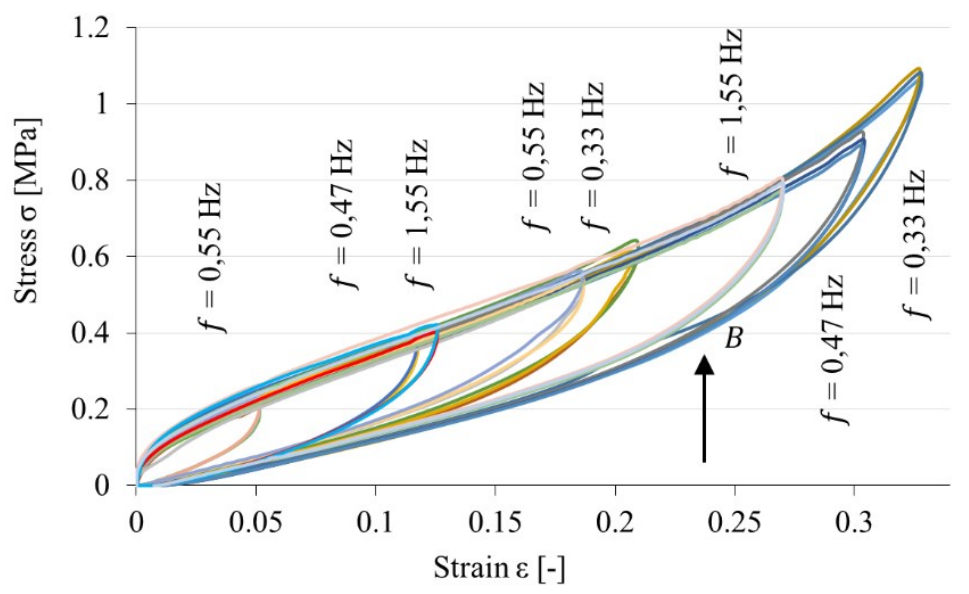

Fig. 8. A set of hysteresis loops determined as a result of the conducted tests

The established values of the storage modulus $E^{\prime}$ and loss modulus $E^{\prime \prime}$ were presented on diagrams 9 and 10. The analysis of the presented characteristics indicates an increase of both moduli as a function of magnetic induction $B$. The apparent increase in rigidity of the tested samples is caused by interactions between the ferromagnetic material molecules in the 
composite and the magnetic field. The composite strain causes molecules to form such a position characterized by minimal energy. Therefore, some amount of work is to be supplied to the system, resulting in an increase of required force to achieve the same degree of strain for the growing value of magnetic induction.

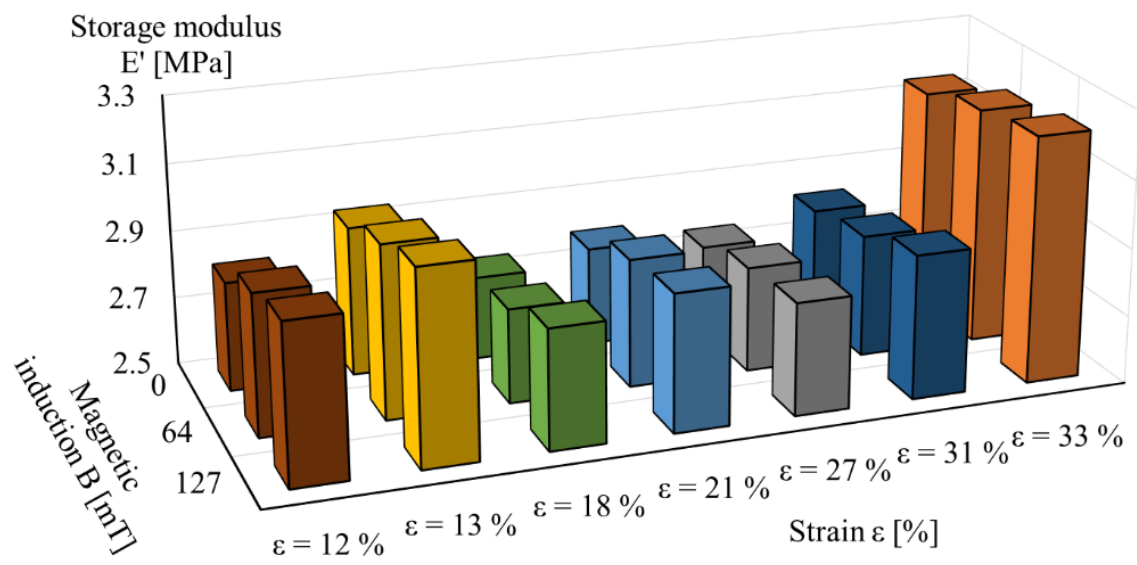

Fig. 9. Change in storage modulus value $E^{\prime}$ as a function of magnetic induction $B$ for different strain values $\varepsilon$

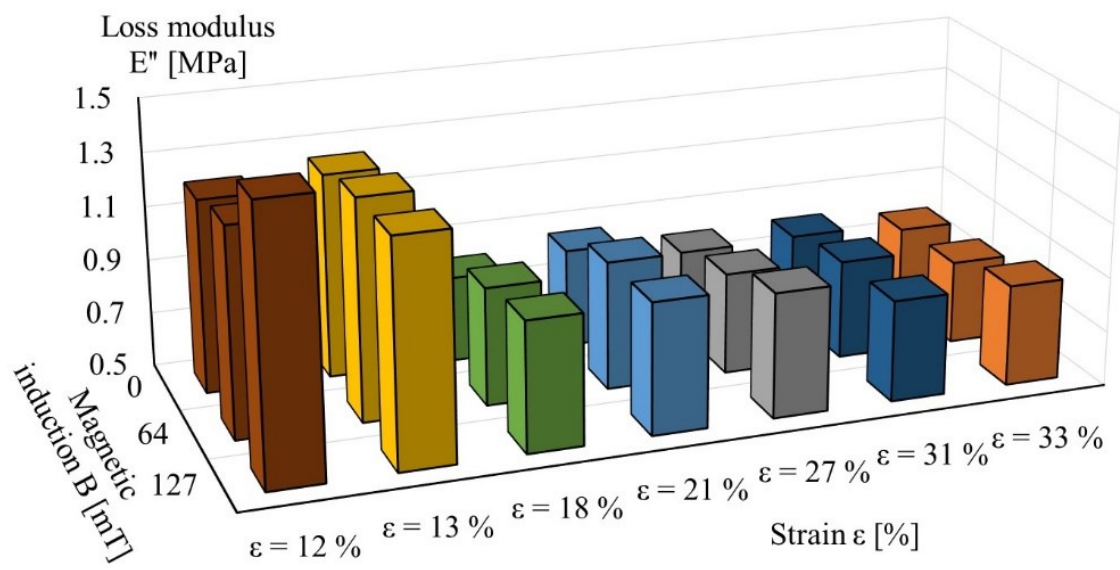

Fig. 10. Change in loss modulus value $E^{\prime \prime}$ as a function of magnetic induction $B$ for different strain values $\varepsilon$

The registered change in dissipated energy $W$ and elastic energy $\Delta W$ for tested samples is provided on Fig. 11. The values of both moduli increase along with the induction of the magnetic field. However, the relative damping value $\psi$ calculated based on these values is decreasing. The area of the triangle $\mathrm{ABC}$ (Fig. 7), related with elastic energy $\Delta W$ increases together with the angle of inclination of individual hysteresis loops in relation to the strain axis of the assumed coordinate system. This growth is an effect of interaction between the magnetic field and ferromagnetic particles, as described earlier. The area of hysteresis loop in relation to dissipated energy $W$ is growing together with the magnetic induction value $B$. It is further dependent on the strain amplitude $\varepsilon_{0}$. The observed results exhibit strongly progressive characteristics. It depends on the maximum strain value and results from 
the change in sample cross-section due to applied load. It is most likely that this phenomenon causes a decrease in relative damping value $\psi$, despite the increase in the value of dissipated energy $W$ and elastic energy $\Delta W$ being caused by the magnetic field. This is because the increase of the entire area of hysteresis loop exhibits faster growth than the growth rate of the $\mathrm{ABC}$ triangle (Fig. 7) for the tested samples. Therefore, one needs to conclude that damping in magnetorheological elastomers under compressive stress depends both on magnetic induction $B$, but is also very strongly dependent on the strain amplitude $\varepsilon_{0}$. This influence must not be overlooked when designing machines utilizing magnetorheological elastomers for vibration damping and dissipation.

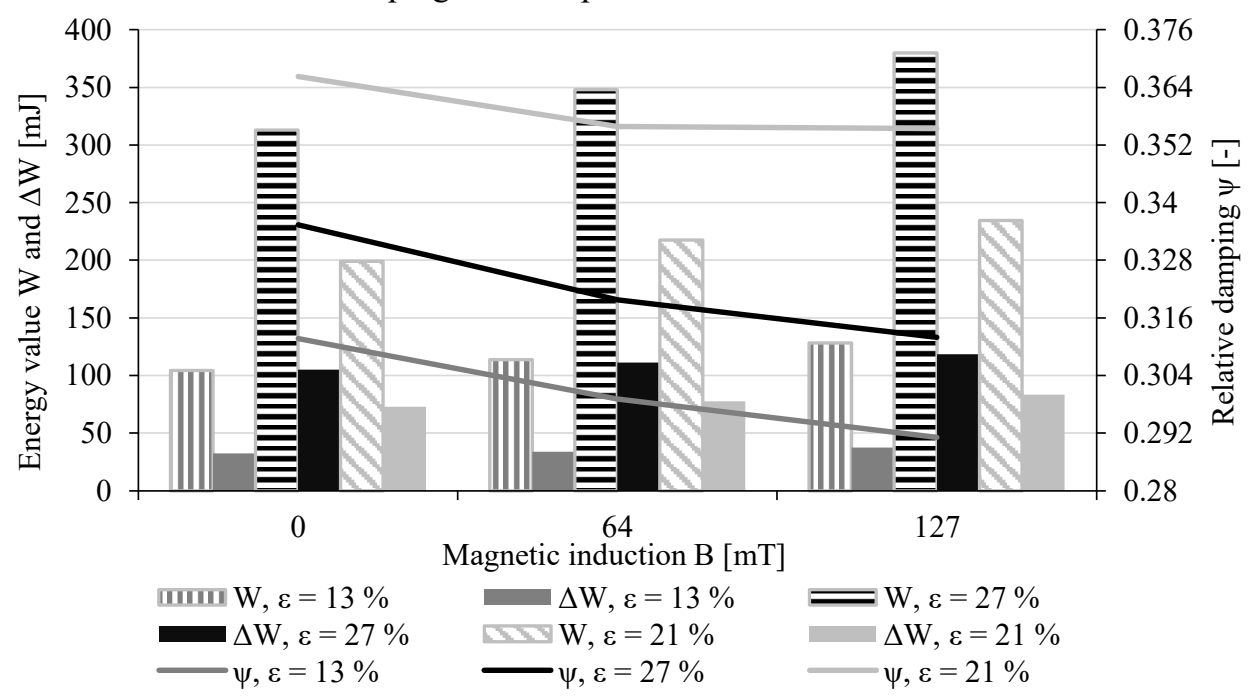

Fig. 11. Selected diagrams for change of dissipated energy $W$ and elastic energy $\Delta W$ together with relative damping $\psi$ for the tested samples

\section{Conclusions}

As a result of the described works, a testing station for studying damping in magnetorheological elastomers under compressive stress was designed and constructed. This allowed to carry out tests on a physical object. The results of the discussed study were used as a basis for establishing a number of material properties. Changes occurring in the mechanical hysteresis loops of magnetorheological elastomers caused by an increase in strain amplitude $\varepsilon_{0}$ and magnetic induction $B$ were identified. The growth of modulus of storage $E^{\prime}$ and loss $E^{\prime \prime}$ was calculated as a function of parameters of applied magnetic field on which the structure of Kelvin-Voigt rheological model is based. It is used to describe the properties of such materials which exhibit both elastic and viscous characteristics. Further, a change of value of dissipated energy $W$ and elastic energy $\Delta W$ was calculated for individual hysteresis loops registered during the experiments. Based on the above, the characteristics of change in the relative damping $\psi$ were identified for the studied composite material. Consequently, the possibility to utilize magnetorheological elastomers in machine construction was identified. This applies in particular to applications which call for control via magnetic field, the possibility to absorb and dissipate mechanical energy. 


\section{References}

1. Sun S. S., Chen Y., Yang J., Tian T. F., Deng H. X., Li W. H., Du H., Alici G. The development of an adaptive tuned magnetorheological elastomer absorber working in squeeze mode. Smart Materials and Structures, 23, 075009, 1-8 (2014)

2. Deng H., Gong X. Application of magnetorheological elastomer to vibration absorber. Communications in Nonlinear Science and Numerical Simulation, 13, 9, 1938-1947 (2008)

3. Liao G. J., Gong X. L., Kang C. J., Xuan S. H. The design of an active-adaptive tuned vibration absorber based on magnetorheological elastomer and its vibration attenuation performance, Smart Materials and Structures, 20, 7, 1-10 (2011)

4. Hoang N., Zhang N., Du H. An adaptive tunable vibration absorber using a new magnetorheological elastomer for vehicular powertrain transient vibration reduction, Smart Materials and Structures, 20, 1, 1-11 (2010)

5. Hoang N., Zhang N., Li W. H., Du H. Development of a torsional dynamic absorber using a magnetorheological elastomer for vibration reduction of a powertrain test rig, Journal of Intelligent Material Systems, vol. 24, pp. 2036-2044. (2013)

6. Mitsumata T., Ohori S. Magnetic Polyurethane Elastomers With Wide Range Modulation of Elasticity, Polymer Chemistry, vol. 2, is. 5, pp. 1063-1067. (2011)

7. Kukla M., Górecki J., Malujda I., Talaśka K., Tarkowski P. The Determination of Mechanical Properties of Magnetorheological Elastomers (MREs), Procedia Engineering, 177, 324-330 (2017)

8. Wałęsa K., Malujda I., Talaśka K. Butt Welding of Round Drive Belts, Acta Mechanica et Automatica, 12, 2, 115-126 (2018)

9. Boczkowska A., Awietjan S. Microstructure and Properties of Magnetorheological Elastomers, Advanced Elastomers - Technology, Properties and Applications, chapter 6, InTech, 147-180 (2012)

10. Pękalak M., Radkowski S. Gumowe elementy sprężyste. Państwowe Wydawnictwo Naukowe, ISBN 83-01-08036-1, Warszawa (1989) 